\title{
La réfutation dans les sciences historiques
}

\section{Claude Grignon}

\section{OpenEdition \\ Journals}

Édition électronique

URL : http://journals.openedition.org/ress/131

DOI : 10.4000/ress. 131

ISSN : 1663-4446

Éditeur

Librairie Droz

Édition imprimée

Date de publication : 1 septembre 2008

Pagination : $35-53$

ISBN : 978-2-600-01242-3

ISSN : 0048-8046

Référence électronique

Claude Grignon, "La réfutation dans les sciences historiques 》, Revue européenne des sciences sociales [En ligne], XLVI-142 | 2008, mis en ligne le 01 septembre 2011, consulté le 21 avril 2019. URL : http:// journals.openedition.org/ress/131 ; DOI : 10.4000/ress.131 


\section{CHAPITRE II}

\section{LA RÉFUTATION DANS LES SCIENCES HISTORIQUES}

Les sciences de l'homme, du moins les plus générales d'entre elles, doivent donc, en accord avec Popper, soumettre leurs idées à la «falsification », c'est-àdire, en bon français, à la réfutation ${ }^{1}$ et, contre Popper, ou plutôt contre le popperisme, renoncer à la croyance selon laquelle la démarche hypothético-déductive est supérieure à l'induction et constitue en fait la seule démarche vraiment scientifique. Au lieu d'aller de la théorie à la réalité (top down), de se placer d'emblée au niveau le plus abstrait et le plus général, de partir d'une idée de principe pour «retrouver »la réalité et la mettre en forme, il leur faut, à l'inverse, se placer pour commencer au niveau phénoménologique, partir de l'observation et de la description des faits pour assurer l'adéquation des entités théoriques qu'elles essaient d'élaborer à la réalité dont elles s'occupent ${ }^{2}$. Il faut combiner ces deux principes pour échapper à l'alternative d'une sociologie (ou d'une ethnologie, ou d'une histoire) purement spéculative, pseudo-théorique et pseudo-scientifique, et d'une sociologie sans idées, réduite à une méthodologie routinière et aveugle, retombée dans l'illusion de la description pure. Pour en finir avec les généralités qui les condamnent à rester en dehors et en deçà de la science, les sciences de l'homme doivent cesser de croire qu'on peut tout expliquer en partant d'une idée-force, d'un parti-pris interprétatif; mais, sous peine de renoncer à être des sciences, elles ne peuvent rester au niveau «concret» des prétendues «lois phénoménologiques », se contenter de collectionner les observations, d'accumuler des «objets de mémoire» dépourvus de signification. Elles doivent elles aussi s'efforcer de «prendre le monde, - c'est-à-dire l'ordre humain - dans leurs filets » pour le rendre plus intelligible; elles ne peuvent s'abstenir d'ordonner les faits pour en faire des «objets de raison», et pour cela elles doivent les comparer, les classer, trouver des ressemblances et des régularités, généraliser et abstraire. Il faut donc qu'elles continuent à imaginer, à inventer, à avoir des idées et à jouer avec elles, au besoin à en emprunter, bref à spéculer, comme les sciences de la nature et les mathématiques ne cessent de le faire; mais pour qu'elles puissent continuer à spéculer, il faut qu'elles puissent sortir de la spéculation, en transformant leurs assertions en questions, leurs idées en conjectures réfutables.

1 Popper lui-même identifie «falsifiability» et «refutability». Unended Quest, op. cit., p. 43.

2 Toutes les sciences sans doute ont recours à la déduction. Mais selon que l'on en part ou que l'on y vient, elle ne joue pas le même rôle dans la recherche; dans le cas des sciences historiques et observationnelles, on passe de l'induction à la déduction, et mieux vaut tard que trop tôt (cf. infra, chap. III, 4). 


\section{L'OBJECTION RÉDUCTIONNISTE}

Ces deux règles, réfutation et théorisation «bottom up», peuvent sembler incompatibles entre elles ${ }^{3}$. Les énoncés des sciences historiques ne seraient pas réfutables; plus exactement, comme ils ne seraient pas absolument réfutables, ils ne seraient absolument pas réfutables. C'est la position de J.-C. Passeron: «Seuls les énoncés universels au sens strict, ou 'énoncés-à-propos-de-tous' comme les nomme Popper, répondent aux exigences logiques de la mécanique falsificatrice, parce que, portant affirmation sur un nombre illimité de cas, ils peuvent être démentis par un seul énoncé existentiel singulier, alors que le démenti d'une proposition 'numériquement universelle' ouvre inévitablement, par-delà sa réfutation formelle, la question du remaniement des coordonnées spatio-temporelles qui pourrait la préserver de la réfutation. Cette seule différence suffit à exclure que n'importe quelle proposition de théorie sociologique soit falsifiable au sens de Popper dès lors qu'on applique rigoureusement la définition de la falsifiabilité donnée par Popper lui-même» (Passeron 1991: 378) ${ }^{4}$. C'est réserver la réfutabilité, et du même coup la scientificité, aux seules sciences nomothétiques, à l'exclusion non seulement des sciences de l'homme, mais de l'ensemble des sciences de la nature dont les énoncés sont contextuels, ou du moins non universels, puisqu'ils portent sur un ordre de phénomènes uniques et localisés (par exemple la vie sur la terre). Si l'on recherche les faits qui vont dans le sens d'une thèse, et si par extraordinaire il n'en vient pas immédiatement à l'esprit, s'il ne s'en présente pas dans le voisinage immédiat du sujet traité, on peut toujours affirmer qu'on en trouverait pourvu qu'on «élargisse le contexte de validité» par un «raisonnement naturel qui est de l'ordre de la composition argumentative» (c'est-à-dire de la rhétorique, sinon de la sophistique). Mais c'est pour le coup qu'il faut être strictement poppérien: si l'on se met à la recherche des faits capables d'infirmer (et non de confirmer) un énoncé, il suffit, dans les limites du contexte pour lequel une proposition théorique prétend valoir, de trouver « un seul énoncé existentiel singulier» incompatible avec les conséquences et les prédictions de cette proposition pour que celle-ci soit réfutée; on se retrouve alors dans le cas des théories des sciences nomothétiques, dont on n'est jamais sûr qu'elles sont vraies, mais seulement assuré que provisoirement, sous réserve de la présentation d'un démenti factuel décisif, elles ne sont pas fausses. Dès lors que l'on considère le contexte d'une proposition comme un «contexte d'invalidation», et non plus comme un « contexte de validité », l'élargissement du contexte, non seulement possible, mais nécessaire pour généraliser progressivement, par comparaisons successives entre des «configurations historiques singulières » (Weber), tourne à l'avantage du réfutateur, puisqu'il augmente les chances de rencontrer des faits contrariants;

3 Le rejet de la réfutation est la pierre angulaire du relativisme; cf. entre autres, l'apologie, par B. Latour, du «principe de Stengers», par opposition au «principe de Popper» qui «conserve tous les montages expérimentaux, fussent-ils remplit d'artefacts, où des êtres réduits a quia répondent par oui ou par non» (La recherche, 301, Septembre 1997). L'impossibilité de distinguer le vrai du faux, érigée en principe, est vitale pour les intellectuels littéraires, que la réfutation réduit au silence.

4 J.-C. Passeron, Le raisonnement sociologique, l'espace non-poppérien du raisonnement naturel, Paris, Nathan, 1991, p. 378. 
loin de «protéger» les propositions théoriques des sciences historiques de la réfutation, le «remaniement des coordonnées spatio-temporelles» ne cesse au contraire de les y exposer.

La conception restrictive de la réfutation à laquelle se réfère J.-C. Passeron se rattache à un réductionnisme philosophique qui hiérarchise les sciences par ordre décroissant de scientificité, des sciences physico-chimiques aux «sciences morales» ou «humaines», des sciences formelles, capables d'énoncer des lois universelles, aux sciences historiques, des sciences expérimentales aux sciences de l'observation. Comme philosophe (mais pas comme mathématicien), Cournot plaçait déjà les sciences «abstraites», «mathématiques» au sommet de cette hiérarchie, l'histoire et l'archéologie se retrouvant avec l'astronomie («l'histoire du ciel »), la géologie, la botanique, et la zoologie dans la «série cosmologique et historique», par opposition à la «série théorique» des connaissances humaines (Cournot 1973 [1872]: 5, 10) ${ }^{5}$. Fondé sur l'opposition entre «science pure et science empirique», «sciences de pur raisonnement et sciences d'observation», le système des sciences d'E. Goblot constitue une expression particulièrement nette d'un programme réductionniste fort, qui érige les mathématiques en idéal de scientificité; pour Goblot, la science ultime est une forme pure, universelle, qui peut s'appliquer indifféremment à tous les phénomènes parce qu'elle est indifférente aux contenus:

L'ensemble de la science (...) paraît se diviser en trois groupes: sciences mathématiques, sciences physiques et naturelles, sciences morales. (...) Les sciences morales, presque aussi anciennes que les autres, sont beaucoup moins avancées: leurs plus précieuses acquisitions ne revêtent que d'une manière imparfaite les caractères de la science; elles n'ont encore fixé ni leurs objets, ni leurs principes, ni leurs méthodes. Mais elles prétendent devenir un jour aussi positives, aussi précises, aussi rigoureusement démonstratives que le sont aujourd'hui les sciences physiques et naturelles (...) Il nous reste à montrer

5 «Il en est (...) de l'histoire des peuples comme de l'histoire de la Nature, qu'il ne faut pas confondre avec la science de la Nature, parce qu'elles ont principalement pour objet l'une des lois, l'autre des faits, mais des faits qui peuvent acquérir une si grande proportion, avoir des conséquences si vastes et si durables, qu'ils nous paraissent avoir et qu'ils ont effectivement la même importance que des lois. La raison n'en conçoit pas moins la différence radicale des lois et des faits; les uns valables en tout temps, en tout lieu, par une nécessité qui tient à l'essence permanente des choses; les autres amenés par un concours de faits antérieurs, et déterminant à leur tour les faits qui doivent suivre». A. A. Cournot, Considérations sur la marche des idées et des événements dans les temps modernes, Paris, Vrin, 1973 [1872], pp. 5, 10. Comme mathématicien, Cournot est conduit au contraire à relativiser l'opposition entre la loi et le fait, et à mettre l'accent sur la continuité des sciences: «Et d'abord, est-il vrai que la science n'ait pour objet que des vérités immuables et des résultats permanents? En aucune façon. (...) Les types spécifiques de la nature sauvage et libre, dont on n'a pas jusqu'ici constaté la variabilité depuis l'origine des temps historiques, pourraient bien être sujets à de lentes modifications, qui au fond ne nuiraient pas plus à la dignité des sciences naturelles que les lentes perturbations du système planétaire (...) ne nuisent à la perfection de l'astronomie. Les rapides changements que le temps apporte dans les faits qui sont du ressort de l'économie sociale, en rendant plus difficile l'étude des sciences économiques dans ce qu'elles ont de positif et de déterminable par l'observation, ne leur enlèvent pas leur caractère de sciences; et en un mot rien n'exige que les objets d'une théorie scientifique soient fixes, invariables, appropriés à tous les temps et à tous les lieux ». «Rien de plus inégal que le degré de généralité des faits sur lesquels portent les sciences, d'ailleurs susceptibles au même degré de l'ordre et de la clarification qui constituent la perfection scientifique». A. A. Cournot, Essai sur les fondements de nos connaissances et sur les caractères de la critique philosophique, Paris, Vrin, 1975 [1851], pp. 360, 362, 363. 
que les sciences de la nature, présentement expérimentales et inductives, tendent à devenir, au terme de leur développement, conceptuelles et déductives, et que, si jamais elles parviennent à leur achèvement, elles seront, comme les mathématiques, indépendantes de la réalité de leurs objets (Goblot 1922: 18, 29) ${ }^{6}$.

Dès lors qu'on se place dans cette perspective, on adopte implicitement une conception purement logique de la réfutation et plus généralement de la preuve; de ce point de vue, c'est en effet l'assertion la plus formelle, «l'assertion mathématique qui est vraie ou non dans un système axiomatique donné $»^{7}$, qui est la seule réfutable en principe. C'est oublier que la «mécanique falsificatrice» ne tourne pas à vide, et que ses «exigences logiques» sont inséparables de ses exigences empiriques. Que la réfutation soit pour finir un jugement qui décide absolument du faux sinon du vrai n'implique pas qu'elle soit exclusivement affaire de raisonnement. L'administration de la preuve exige la présentation de faits décisifs; pour suivre et contrôler un raisonnement propositionnel, pour rejeter ou accepter les conclusions d'une hypothèse, il faut enquêter et constater, procéder à des investigations qui apparentent l'exploration scientifique à l'enquête policière ${ }^{8}$, recueillir les preuves factuelles, les evidences, «the data on which a conclusion or judgment may be based » ${ }^{9}$. C'est parce que les objets inventoriés par l'enquête sont des objets singuliers, désignés par des noms propres, donnant lieu à une connaissance d'objet (et non à une connaissance propositionnelle), que leur présence (ou leur absence) peut «lever le doute» et décider, sans ambiguiité, du sort d'une conjecture. A l'inverse, l'idée que les contenus sont indifférents parce qu'interchangeables entraîne et entretient l'indifférence quant aux contenus.

En faisant oublier que la réfutation est d'abord une confrontation avec la réalité et en la réduisant à un contrôle logique réservé par principe aux sciences «indépendantes de la réalité de leurs objets », la dérive formaliste la rend paradoxalement accessible et indulgente aux généralités spéculatives; en effet, une idée-force, un parti-pris interprétatif ne se distinguent pas, sous ce rapport, d'une axiomatique: il leur suffit d'être développés de manière systématique et cohérente pour être validés. Les généralités dont la culture générale est faite ressemblent aux lois générales en ce qu'elles sont elles aussi, à leur manière, indifférentes aux

6 E. Goblot, Le système des sciences, le vrai, l'intelligible et le réel, Paris, Armand Colin, 1922, pp. 18, 29. Le système de Goblot, sa philosophie de l'histoire des sciences sont également réductionnistes en ce qu'ils supposent que la complexité dont les degrés croissants distinguent les différents ordres de réalité et les différentes sciences n'est qu'une apparence, une illusion qui se dissipe à mesure que la science progresse et s'unifie en rejoignant le modèle idéal des mathématiques.

7 J.-L. Puget, «Les relations de l'astrophysique et de la cosmologie avec l'observation: critères de vérité », in J.-P. Changeux, La vérité dans les sciences, Paris, O. Jacob, 2003, pp. 187-199.

8 «Le médecin épidémiologiste se fait détective pour identifier son suspect et le suivre à la trace par les voies qu'il emprunte». A. Fagot-Largeault, La recherche étiologique, chemins causals, histoires, influences, mécanismes, ontologie causale, Universidade Federal de Santa Catarina, Florianopolis, avril 2004.

9 «La preuve est, en général, un raisonnement; mais non pas toujours; elle peut consister en une présentation de fait qui lève le doute. De là vient que ce mot, en un sens pour ainsi dire matériel, s'applique aussi au fait, au document qui prouve quelque chose.» A. Lalande, op. cit., article «Preuve». 
contenus. Mais la ressemblance est trompeuse: à la différence et à l'inverse des lois, qui sont d'autant plus générales qu'elles sont plus riches de contenu (que la quantité et l'hétérogénéité des phénomènes auxquels elles s'appliquent sont plus grandes), les idées générales ne paraissent telles que parce qu'elles sont suffisamment vagues; plus elles sont indéfinies et indéfinissables, moins l'on sait et moins l'on peut savoir ce qu'elles désignent, et plus elles semblent pouvoir s'appliquer indifféremment à tout, c'est-à-dire en fait à n'importe quoi. Cette ressemblance n'en contribue pas moins à entretenir la confusion entre théorie scientifique et pseudo-théorie, et peut-être aussi la fascination que les sciences les plus formelles exercent sur les producteurs d'idées générales.

Le positivisme dont le rationalisme de Goblot est empreint est sans doute intellectuellement démodé; mais la croyance dans la hiérarchie des sciences, alimentée par la capacité inégale de celles-ci à déboucher sur des applications et sur des techniques, est passée dans l'opinion commune (comme en témoigne la popularité de l'opposition entre «sciences dures» et «sciences molles»). Elle persiste dans la philosophie des sciences (du moins dans celle des sociologues venus de la philosophie), et aussi, bien qu'elle corresponde de moins en moins à l'état des sciences, dans la philosophie spontanée et inconsciente des scientifiques ${ }^{10}$. Bien qu'on sache maintenant que les lois de la physique newtonienne ne sont vraies que dans certaines limites, c'est-à-dire dans un contexte donné ${ }^{11}$, l'idéalisation de la notion de loi universelle continue à fonder l'idée de la supériorité des sciences nomothétiques sur les sciences historiques. De même, l'idée de la supériorité de la démarche hypothético-déductive demeure fermement ancrée, jusque dans les sciences de l'homme, et pas seulement chez les économistes.

\section{EXPÉRIMENTATION ET OBSERVATION}

L'idée de la supériorité de l'expérimentation sur l'observation, et, en conséquence, de la supériorité des sciences expérimentales sur les sciences observationnelles continue elle aussi à être communément admise. L'idéalisation de l'expérimentation repose sans doute pour une part sur l'assimilation de celle-ci à l'expérience cruciale, instantia crucis, qui permet en effet, comme son nom l'indique, de trancher entre deux hypothèses exclusives l'une de l'autre. C'est oublier que la plupart des expériences ne sont pas cruciales, et qu'il existe des observations décisives; c'est oublier aussi qu'il existe des expériences «pour voir», c'està-dire des expériences aveugles, et qu'il ne faut pas confondre l'observation pour l'observation, au hasard, sans guide, avec l'observation scientifique, sélective,

${ }^{10}$ Sur le suprématisme («l'arrogance ») de certains physiciens, cf. E. Mayr, Histoire de la biologie, op. cit., p. 45.

11 «On sait bien qu'aux vitesses proches de la lumière l'accélération d'une particule déviera considérablement de la mécanique newtonienne. Un physicien dira communément que la mécanique newtonienne est vraie avec une certaine précision tant qu'on se trouve dans une certaine gamme de vitesses. Le critère de vérité est donc qualifié par une quantification de précision et quant aux conditions de sa validité ». J.-L. Puget, art. cit. 
raisonnée et préméditée ${ }^{12}$. Par ailleurs, on passe graduellement des sciences qui peuvent monter à volonté des expériences cruciales à celles qui doivent se contenter d'observer les situations expérimentales que présente le cours des phénomènes qu'elles étudient; comme le montre, entre quantité d'autres, l'expérience de Michelson, ces expérimentations indirectes peuvent être aussi décisives que des expériences provoquées. L'opposition entre sciences expérimentales et sciences de l'observation ne correspond ni à l'opposition entre sciences de la nature et sciences de l'homme, ni, à l'intérieur des sciences de la nature, à l'opposition entre sciences physiques et sciences de la vie; la médecine est expérimentale, à des degrés variés, dans les limites que lui impose la morale collective, l'astrophysique observe (Bernard 1966 [1865]) $)^{13}$. Les sciences physiques et les sciences de l'homme se situent sans doute aux deux extrémités opposées de ce continuum. Dans les sciences de l'ordre physico-chimique, l'observation s'insère dans une démarche hypothético-déductive pour répondre, comme l'expérimentation, aux questions posées par des théories qui la précèdent et qui la suscitent; c'est le cas, par exemple, en cosmologie, où elle confirme ou infirme les prédictions des «modèles d'univers» construits à partir des lois de la physique fondamentale (Puget 2003) ${ }^{14}$. Les astrophysiciens peuvent anticiper avec certitude les situations expérimentales qui s'offrent à eux; il n'en va pas de même pour les sociologues

12 «En réalité, de nombreux articles s'appuyant sur des expériences faites sans Fragestellung adéquate sont plus descriptifs que la plupart des publications non-expérimentales, en biologie évolutive». E. Mayr, Histoire de la biologie, op. cit., p. 43.

${ }^{13}$ Comme on peut s'y attendre, C. Bernard affirme la supériorité de la médecine expérimentale sur la médecine observationnelle: «On le voit, la médecine expérimentale n'exclut pas la médecine clinique d'observation; au contraire, elle ne vient qu'après elle. Mais elle constitue une science plus élevée et nécessairement plus vaste et plus générale (...) En un mot, je considère l'hôpital seulement comme le vestibule de la médecine scientifique; c'est le premier champ d'observation dans lequel doit entrer le médecin, mais c'est le laboratoire qui est le vrai sanctuaire de la science médicale; c'est là seulement qu'il cherche les explications de la vie à l'état normal et pathologique au moyen de l'analyse expérimentale». Mais la «méthode d'investigation ne distingue pas celui qui observe de celui qui expérimente». Le raisonnement expérimental «sera absolument le même dans les sciences d'observation et dans les sciences expérimentales». L'astronome, «forcé de se borner à l'observation», n'en raisonne pas moins comme les expérimentateurs, «parce que l'expérience acquise implique partout jugement et comparaison entre deux faits liés dans l'esprit par une idée». Ce qui distingue les sciences expérimentales des sciences d'observation, c'est « la puissance de l'investigateur d'agir sur les phénomènes». Une science d'observation est donc une «science passive». «Dans les sciences d'expérimentation, l'homme observe, mais de plus il agit sur la matière (...) et provoque à son profit l'apparition de phénomènes, qui sans doute se passent toujours suivant les lois naturelles, mais dans des conditions que la nature n'avait souvent pas encore réalisées. A l'aide de ces sciences expérimentales actives, l'homme devient un inventeur de phénomènes, un véritable contremaître de la création». Introduction à la médecine expérimentale, op. cit. , pp. 205, 206, 44, 46, 47, 48.

14 «Ces questions [l'homogénéité à grande échelle de l'Univers, sa géométrie, sa stationnarité ou son évolution dans le temps] illustrent bien comment la théorie tend souvent, en cosmologie (...) à précéder les observations, ce qui n'est pas le cas dans les disciplines s'attachant à décrire des phénomènes complexes». Ainsi, «c'est bien la mise en évidence récente par deux mesures différentes d'un écart aux relations prédites par le modèle sans constante cosmologique qui a conduit à réintroduire un terme de 'constante cosmologique' dans les équations de la dynamique de l'Univers. (...) La découverte de ce rayonnement (le rayonnement cosmologique) en 1965 par Penzias et Wilson et la démonstration par John Mather de son caractère 'planckien' sont sans aucun doute l'élément qui a transformé le modèle controversé du big-bang en modèle standard de la cosmologie.» J.-L. Puget, art. cit. 
confrontés aux hasards de l'histoire (ni du reste pour les géophysiciens, qui peuvent prédire le lieu mais non le moment d'une éruption volcanique ou d'un tremblement de terre). Mais les opportunités que présente le cours des événements ne sont pas toujours imprévisibles, et elles s'annoncent parfois suffisamment à l'avance pour qu'on s'y prépare (c'était par exemple le cas du «passage à l'euro»). Les sociologues peuvent mettre à profit les «expériences naturelles» que constituent les catastrophes historiques:

\begin{abstract}
Les remarques précédentes [sur la psychologie du témoignage] s'appliquent aux expériences proprement dites, œuvres artificielles de l'ingéniosité humaine. Ce qui nous limite, en l'espèce, ce sont les bornes mêmes qui s'imposent à l'action d'un savant, bien incapable évidemment dans son laboratoire de modifier la constitution de la société ou de créer de grandes émotions communes. Mais voici qu'il s'est produit dans ces dernières années une sorte de vaste expérience naturelle. On a le droit en effet de considérer comme telle la guerre européenne: une immense expérience de psychologie sociale d'une richesse inouïe. Les conditions nouvelles d'existence, d'un caractère si étrange, avec des particularités si accentuées, où tant d'hommes à l'improviste se sont trouvés jetés - la force singulière des sentiments qui agitèrent les peuples et les armées - tout ce bouleversement de la vie sociale, et, si l'on ose ainsi parler, ce grossissement de ses traits, comme à travers une lentille puissante, doivent, semble-t-il, permettre à l'observateur de saisir sans trop de peine entre les différents phénomènes les liaisons essentielles. Sans doute ne peut-il pas, comme dans une expérience au sens ordinaire du mot, faire varier lui-même les phénomènes pour mieux connaître les rapports qui les unissent; qu'importe, si ce sont les faits eux-mêmes qui montrent ces variations, et avec quelle ampleur! (Bloch 2006 [1921]: 300) ${ }^{15}$
\end{abstract}

Dans la mesure où elle permet d'établir des relations entre des faits et de les faire varier à volonté, l'étude statistique peut être considérée sinon comme un équivalent, du moins comme un substitut de l'expérimentation; le raisonnement du sociologue statisticien est un raisonnement expérimental (Simiand 1922) ${ }^{16}$. Par ailleurs les interventions du sociologue, comme celles du politique ou du journaliste, sont autant d'expérimentations involontaires; c'est le cas de toutes les situations d'enquête, lorsqu'on fait des interviews, lorsqu'on administre un questionnaire, quand on publie les résultats d'un sondage, etc. A l'échelle des groupes restreints et des interactions, qui est, il est vrai, celle de la psychologie sociale plutôt que de la sociologie générale, il est donc possible de monter de véritables expériences. En posant des questions inattendues, en adoptant des comportements inadaptés et importuns, le sociologue suscite des réactions et des sanctions révé-

${ }^{15}$ M. Bloch, «Réflexions d'un historien sur les fausses nouvelles de la guerre», Revue de synthèse historique, t. 33, 1921, pp. 13-35, in M. Bloch, L'Histoire, la Guerre, la Résistance, op. cit., p. 300 .

16 «Il y a expérience partout où et seulement là où il y a disposition des faits telle que l'esprit de l'homme puisse tirer une relation entre ces faits ». «Sans doute il y a (...) cette première différence (...) que la simplification des données, l'isolement d'un élément, et la recherche d'une relation avec un autre facteur séparé sont réalisés par le savant, dans l'un des cas, au moyen d'opérations matérielles, physiques, dans l'autre, au moyen d'opérations non physiques, intellectuelles. Mais est-ce donc le moyen, - matériel ou intellectuel — de l'opération de l'homme qui est l'essentiel de l'expérience, et non pas l'objet même de cette opération?» F. Simiand, Statistique et Expérience, remarques de méthode, Paris, Marcel Rivière, 1922, pp. 11, 10. Sur les limites que le caractère immatériel des faits sociaux impose à «l'expérimentation» statistique, cf. infra, chap. IV, 1. 
latrices. Ce genre de provocation risque sans doute de tomber dans le canular édifiant, conformément au modèle de l'artiste bohème, qui va toujours plus loin dans les bravades destinées à choquer le bourgeois, ou du journaliste farceur, spécialiste des blagues dénonciatrices. On peut toujours s'amuser à apporter son manger à la Closerie des Lilas ou l'Auto-journal à un séminaire de l'EHESS, mais ces expériences pour rire ne sont que des expériences pour voir. Plus sérieusement, on peut questionner, dans la tradition de l'ethnométhodologie, les énoncés qui vont de soi, sur lesquels tout le monde s'accorde, et objectiver ainsi le sens commun propre à un milieu, les conventions et les pactes tacites sur lesquels reposent les échanges et la compréhension entre ses membres. En choquant les enquêtés, le sociologue leur fait extérioriser leur système de valeurs et de croyances implicite et ultime, il force celui-ci à se manifester et, pour ainsi dire, à se matérialiser - une expérience qui peut être dangereuse, «explosive». On peut aussi déclencher des mécanismes de rejet et d'exclusion, «tester» un groupe en faisant varier les caractéristiques sociales (âge, sexe, origine, etc.) des individus qu'on lui présente et qu'on lui demande de recevoir.

\section{LA DÉRIVE INTERPRÉTATIVE}

En outre il n'est pas d'expérimentation sans observation; un expérimentateur est toujours un observateur, et pas seulement un opérateur. L'observation ellemême repose sur une série d'interprétations, en commençant par la reconnaissance et l'identification initiales de ce que l'on perçoit. Les risques de dérive interprétative sont sans doute plus grands dans les sciences de l'homme que dans les sciences de la nature; mais celles-ci ne sont pas à l'abri des erreurs d'interprétation, comme en témoigne le développement des méthodes statistiques destinées à contrôler l'observer variability en biologie, notamment dans l'interprétation de l'imagerie médicale, ou en éthologie animale ${ }^{17}$. L'allodoxia géocentrique des «canaux de Mars » illustre la force d'un préjugé interprétatif qui oriente et fausse dès le départ l'observation, oblige à voir ce qui le confirme, empêche de voir ce qui le contrarie. Lorsqu'elles succombent à cette obsession interprétative qui conduit l'observateur à voir partout des signes de ce qu'il veut et croit voir, les sciences les plus exactes ne se distinguent plus des savoirs herméneutiques; l'astronomie elle-même s'apparente à l'astrologie. Mais, à la différence des sciences de l'homme, les sciences de la nature peuvent compter sur les progrès des techniques d'observation pour dissiper les illusions d'optique et les biais interprétatifs suscitées par une théorie; les images de la surface martienne prises par des sondes spatiales en 1965 et en 1969 ont fini par «montrer l'inexistence» des canaux que Schiaparelli avait cru voir au télescope un siècle plus tôt. La possibilité de rejeter

${ }^{17}$ Cf. Wyer P. C. et al. «Tips for learning and teaching evidence-based medicine: introduction to the series [editorial]», CMAJ 2004; 171(4):347-8; Maclure M., Willett W. C. «Misinterpretation and misuse of the kappa statistic». Am J Epidemiol 1987; 126:161-9; Koelemay M. J. et al. «Interobserver variation in interpretation of arteriography and management of severe lower leg arterial disease». Eur J Vasc Endovasc Surg 2001; $21: 417-22$; Conner, RN, «The effect of observer variability on the MacArthur foliage density estimate», Wilson Bull., 102(2), 1990, pp. 341-343. 
le principe interprétatif qui conditionne l'identification des phénomènes dépend du degré auquel celui-ci désigne des evidences, des objets qui s'imposent à l'observation parce qu'ils sont à la fois réels et matériels. C'est le cas pour les canaux, même si la définition commune du canal suppose déjà une modélisation implicite, la sélection des traits pertinents (régularité, aspect rectiligne) qui le distinguent de la rivière; ce l'est aussi pour les vestiges mis au jour par les paléontologues ou par les archéologues. En revanche les faits humains, sociaux, ethnologiques, historiques sont réels sans être matériels: la «société», la «culture», «l'histoire», la «civilisation», «la religion», la «magie», le «politique», sont des abstractions qui échappent à l'observation directe. La naturalisation des faits humains bute sur leur «caractère immatériel» (Durkheim 1967 [1893]: 28) ${ }^{18}$, et sur la confusion spontanée entre réalité et matérialité, qui empêche de les considérer et de les traiter comme des choses.

Le vocabulaire théorique de la sociologie, de l'anthropologie et de l'histoire est donc constitué pour une bonne part par des concepts qui ressemblent beaucoup à des idées générales, et qui se laissent plus ou moins spécifier parce qu'ils manifestent inégalement «leur présence par des effets sensibles » ${ }^{19}$. Il est plus facile, par exemple, de définir l'école que l'éducation, la famille que la classe, la société industrielle que la société dans son ensemble («société» est un «mot primitif», l'équivalent en sociologie de la «vie» en biologie $)^{20}$. Si abstraite qu'elle soit, la notion de marché renvoie plus directement que les notions de valeur, de capital ou de champ à des objets ethnographiques observables et descriptibles. On passe ainsi par degrés des notions les plus représentatives de contenus concrets à des notions de moins en moins définissables et de plus en plus vagues; cette continuité favorise et entretient la confusion entre idées générales et concepts théoriques. Plus les sciences de l'homme ont une vocation générale, et plus elles sont exposées à cette confusion, que la sociologie littéraire ne se prive pas d'exploiter et dans laquelle elle trouve en fait une bonne part de son inspiration. On a parlé ainsi de «société de consommation», de «société d'abondance», de «culture de masse», de «société de spectacle», de «civilisation des loisirs», on parle de «société victimaire», de «culture du risque», de «sociétés post-modernes » voire «post post-modernes», etc. Fondées sur l'association de mots évocateurs, familiers et passe-partout, ces formules disent «quelque chose à tout le monde» parce

${ }^{18}$ E. Durkheim, De la division du travail social, Paris, PUF, 1967 [1893], p. 28.

19 «La solidarité sociale est un phénomène tout moral qui, par lui-même, ne se prête pas à l'observation exacte ni surtout à la mesure. Pour procéder tant à cette classification qu'à cette comparaison, il faut donc substituer au fait interne qui nous échappe un fait extérieur qui le symbolise et étudier le premier à travers le second. Ce symbole visible, c'est le droit. En effet, là où la solidarité sociale existe, malgré son caractère immatériel, elle ne reste pas à l'état de pure puissance, mais manifeste sa présence par des effets sensibles ». E. Durkheim, loc. cit.

${ }^{20}$ «Société, c'est le type même du concept confus, équivoque. Qu'est-ce que Durkheim entend par société, la famille, le public d'un cours, une université, un pays, l'humanité tout entière ? Quelle est celle de ces réalités qu'il baptise société et pourquoi veut-il imposer un choix entre un concept équivoque, faute de définition et une notion qui n'a aucune place dans la science, parce qu'elle est transcendante?». R. Aron, Les grandes doctrines de sociologie historique, op. cit., t. II, Emile DurkheimVilfredo Pareto-Max Weber, p. 100. 
que nul ne sait au juste ce qu'elles veulent dire; selon une recette éprouvée, le malentendu devient alors la condition de l'échange et l'aliment du débat ${ }^{21}$.

Plus ces formules sont vagues, plus elles sont riches en connotations et pauvres en références à la réalité, et plus elles emportent la conviction en faisant appel aux sentiments. Les formules les plus vagues sont aussi les plus propices à l'association spontanée des idées, des mots et des images, dont elles procèdent et qu'elles stimulent (Pareto 1968 [1917]) ${ }^{22}$. Sous peine de casser le ressort de l'invention et de l'imagination on ne peut sans doute s'interdire de jouer librement avec les idées (et donc avec les images et avec les mots). Dans la mesure où elle permet d'essayer des rapprochements et des comparaisons insolites, l'association des idées prépare le regroupement de phénomènes apparemment hétérogènes, qu'on «n'a pas idée», spontanément, de rassembler; elle est indispensable à la production et à la mise au point des concepts abstraits et généraux. Les sciences de l'homme sont donc obligées, comme toutes les sciences, de recourir à l'association des idées, mais cette obligation est dans leur cas particulièrement risquée. Erigée en règle ou en «méthode» pour la «mise en évidence du sens latent d'un matériel», la libre association, qui «consiste à exprimer sans discrimination toutes les pensées qui viennent à l'esprit, soit à partir d'un élément donné (mot, nombre, image d'un rêve, représentation quelconque), soit de façon spontanée», est au principe de l'interprétation libre (Laplanche et Pontalis 1973) 23 $^{23}$ elle est

${ }^{21}$ Voir, entre autres, les sommaires des revues Mouvements ( Classes, exploitation, totem ou tabou?», «Hip-hop, les pratiques, le marché, la politique», «La société saisie par le droit», «Les musulmans dans la modernité»), Tumultes («Temps, mémoire et politique», «La modernité à la trace», «Révolution: entre tradition et horizon», «Le Paria une figure de la modernité »), Sociétés $\left(\mathrm{n}^{\circ}\right.$ 86, «Mélancolie sociale»: «La mélancolie et le tragique sociétal», «La mélancolie au creux de la modernité», «Les mélancolies postmodernes», «La musique metal: des 'éclats de religion' et une liturgie» et, dans les «Excursus heuristiques», «Metal versus Gothic» et «La blandice de Satan. Les satanismes dans le metal») ou Actes de la recherche en sciences sociales ( $n^{\circ} 151-152$, «Sociologie de la mondialisation $», \mathrm{n}^{\circ} 155$, «Le capital militant $)$.

22 «Au contraire, les raisonnements logico-expérimentaux, qui ont leur fondement dans l'observation objective, sont amenés à ne se servir des termes que pour désigner les choses; et par conséquent à les choisir de manière à éviter toute ambiguïté; à les rendre aussi précis que possible. Ces raisonnements engendrent d'ailleurs un langage technique spécial, qui leur permet d'échapper ainsi à l'indétermination du parler courant. Nous l'avons dit déjà: notre intention étant de n'employer que le raisonnement logico-expérimental, nous mettrons tout notre soin à n'user que de mots aussi précis que possible, bien déterminés, et correspondant à des choses, sans équivoques ni ambiguïtés, ou mieux, avec la plus petite erreur possible (...) Les économistes littéraires (...) se complaisent à rechercher ce qu'est la valeur, le capital, etc. On n'arrive pas à leur mettre dans la tête que les choses sont tout et les mots rien. ( ...) Celui qui cherche 'ce qu'est le capital, ce qu'est la valeur, ce qu'est la rente, etc.', montre par cela seul qu'il donne la première place au mot, la seconde à la chose. Le mot capital, par exemple, existe certainement pour lui; il se demande ce qu'il représente et s'efforce de le trouver. En science, on suit une voie opposée à celle-là; c'est-à-dire qu'on s'occupe d'abord de la chose, et qu'ensuite ou lui cherche un nom. On commence par considérer le corps formé par la combinaison de l'oxygène et de l'hydrogène, puis l'on pense à le baptiser. Puisque ce corps se trouve en grande quantité dans la chose mal définie que le langage vulgaire appelle l'eau, on le nomme aussi l'eau; mais ou aurait tout aussi bien pu le nommer autrement, par exemple Lavoisier, ce qui ne changerait rien à la chimie. On dirait simplement que la mer et les fleuves contiennent une grande quantité de Lavoisier. Économistes et sociologues littéraires n'y comprennent rien, parce que l'habitude d'esprit et la culture nécessaires leur font défaut.»V. Pareto, Traité de sociologie générale, op. cit., § 118.

${ }^{23}$ Cf. J. Laplanche et J.-B. Pontalis, Vocabulaire de la psychanalyse (sous la direction de D. Lagache), Paris, PUF, 1973, pp. 228, 229. «L'écrivain Ludwig Borne, que Freud lut dans sa 
donc à l'origine de la dérive herméneutique et des délires d'interprétation dont les sciences de l'homme les plus générales sont menacées quand elles tombent sous l'influence d'un système de pensée personnel (Lanteri-Laura 1964) ${ }^{24}$.

On s'engage dans la voie du verbalisme et des généralités dès que l'on cède à des facilités d'écriture qui sont à l'opposé des exigences de l'esprit scientifique; c'est le cas, entre autres, du procédé qui consiste à supprimer les déterminants, en employant par exemple absolument, à l'infinitif, sans sujet ni complément, des verbes transitifs comme «surveiller», «punir» ou «faire» (lorsqu'on parle de «manières » ou «d'arts de faire»). Cette empoétisation peuple le discours savant d'entités mystérieuses qui tiennent lieu d'abstractions bien construites. M. Mauss déplorait déjà la tendance des sociologues «à composer, à se laisser diminuer, à consentir à être refoulés sur le terrain des généralités concernant la vie sociale»:

Ce rapetissement de la sociologie en sociologie générale a des conséquences extrêmement graves. On l'isole des autres sciences sociales, on lui mesure sa part, on la rend philosophique. Nous voyons non sans anxiété, en Amérique et en Angleterre, se répandre une certaine façon d'écrire et d'enseigner en sociologue. On y considère avec facilité, trop exclusivement, les phénomènes tout à fait généraux de la vie sociale. Même, en fait, et dans certains cas, l'œuvre de certains professeurs en renom se distingue mal d'une sorte de rhétorique brillante, d'une prédication politique et morale, quand elle n'est pas religieuse. Alors, à bon droit, d'excellents esprits réagissent contre ce qu'ils appellent le 'journalisme rationnel', répètent à propos de la sociologie l'épithète de 'philosophie paresseuse' (Mauss 1969: 292) ${ }^{25}$.

La sociologie philosophique dont parle Mauss ressemble beaucoup à la sociologie littéraire dont parle Pareto; l'une et l'autre appartiennent, dans la tradition

jeunesse, recommandait, pour 'devenir un écrivain original en trois jours' d'écrire tout ce qui vient à l'esprit, et dénonçait les effets de l'autocensure sur les productions intellectuelles». Freud en fait mention «dans une note Sur la préhistoire de la technique analytique (Zur Vorgeschichte der analytischen Technik, 1920)».

${ }^{24}$ Cf. G. Lanteri-Laura, «Etude clinique des aspects divers de la paranoïa », Perspectives psychiatriques, $\mathrm{n}^{\circ}$ 7, mars-avril 1964, pp. 19-25. «On emploie la locution de fausseté du jugement pour dire que ces sujets, même quand leur intelligence est de valeur, ne peuvent plus s'en servir quand ils se trouvent eux-mêmes en cause: le doute leur reste aussi étranger que l'autocritique». «Le patient éprouve une perte progressive puis totale de l'expérience vécue, de la contingence: plus rien n'arrive au hasard, tout s'avère porteur d'un sens d'abord caché, puis éclatant (...) Cette exclusion de la contingence est vécue dans la forme de l'interprétation qui devient la manière fondamentale et exclusive dont il appréhende le réel: tout a un sens pour lui, tout veut dire quelque chose (...) l'interprétation tire une conclusion délirante d'une conclusion exacte, elle est vécue, avec une évidence absolue, elle se repère aux thèmes du délire et elle se fonde sur des liens de plus en plus lâches de contiguïté et de comparaison, utilisant parfois des analogies phonétiques et des jeux de mots ».

Comme l'explication de textes, qui est elle-même la version scolaire et laïcisée de l'exégèse, l'analyse de contenu des interviews ou des documents, en sociologie et en histoire, l'analyse des mythes en anthropologie, sont partagées entre l'explication et l'interprétation. Ou bien on s'efforce de reconstituer les conditions et les enchaînements qui ont conduit à la production des représentations que l'on étudie, on cherche, comme dans l'analyse musicale, à en identifier les composants et à en décrire l'organisation; ou bien on s'en inspire, au risque d'ajouter le délire au délire, comme c'est souvent le cas dans l'interprétation des rêves, de la poésie métaphorique ou de la métaphysique allemande.

${ }^{25}$ «Note de méthode sur l'extension de la sociologie», in M. Mauss, Euvres, op. cit., t. III, p. 292. 
française, au genre intermédiaire de la «dissertation littéraire générale $»^{26}$. Les concepts-clé des pseudo-théories sont des concepts indéterminés, qui entretiennent et accroissent la confusion sous couvert de généraliser. «Habitus», par exemple, est «mis pour», et donc «veut dire» indifféremment personnalité, caractère, tempérament, identité, ipséité, attitude, maintien, posture, etc., toutes notions plus ou moins vagues, plus ou moins savantes, plus ou moins construites qu'il faudrait au contraire travailler à préciser et à définir, en les distinguant soigneusement les unes des autres et en examinant leurs relations réciproques. Par le truchement de l'habitus, on va et vient en continu et sans même s'en apercevoir de l'hexis - la manière de se tenir, de se présenter, l'apparence, le corps - à l'ethos - le système de valeurs, les habitudes de pensée, l'esprit -, de l'ethos à l'eidos et à l'essence. On en arrive ainsi à une confusion organisée, systématique, qui clôt la question de l'intériorisation en faisant croire que l'intérieur participe de l'extérieur (et réciproquement). Cette magie interprétative dispense et empêche de se mettre au travail, d'entreprendre et même d'imaginer les enquêtes qui permettraient d'inventorier la diversité des mécanismes sociaux de l'apprentissage et de l'éducation, et de parvenir ainsi, progressivement, à une véritable théorie de la reproduction, ou, plutôt, de l'hérédité, de la génétique sociales. Comme tous les concepts obscurs et hermétiques, l'habitus exige l'initiation et appelle l'exégèse; impossible à dissiper, le mystère qui l'entoure explique sans doute la fascination qu'il exerce sur les sociologues littéraires. Inutile, nuisible même pour la recherche, il s'attire ainsi l'intérêt et la faveur des commentateurs.

Des concepts comme «structure» ou «champ» semblent à première vue moins scolastiques et moins littéraires, parce qu'ils sont empruntés aux sciences de la nature («champ» vient de la physique par l'intermédiaire de la psychologie de la forme, puis de la psychologie sociale, où Pierre Bourdieu l'emprunte à Kurt Lewin); mais une fois arrachées au contexte théorique spécifique dans lequel elles ont été élaborées, des notions telles que «force» ou «tension» perdent le sens spécial que la physique leur a donné et retrouvent leurs connotations primitives ${ }^{27}$. Appliqué aux faits sociaux, le concept de champ se réduit à une métaphore, à un mot d'autant plus évocateur qu'il ne correspond plus à rien de précis. Comme les généralités à la mode, ces abstractions ne semblent pleines de sens que parce qu'elles sont vides de contenu; elles ne paraissent s'appliquer à la totalité des faits sociaux et historiques que parce qu'elles négligent par principe les spécificités, les différences et les variations systématiques qu'il faudrait expliquer. Alors que les succès théoriques reposent, dans les sciences de l'observation, sur une généralisation progressive qui s'efforce de rassembler des phénomènes définis par le plus grand nombre possible de propriétés distinctives, on se situe d'emblée à un niveau de généralité qui ne retient que les similitudes les plus triviales entre les objets.

${ }^{26}$ Sur l'opposition entre «les gens de lettres de la philosophie» [Literatenphilosophen] et les «philosophes sérieux », «élevés à l'école des vrais philosophes de la grande tradition», cf. E. Husserl, La crise des sciences européennes et la phénoménologie transcendantale, Paris, Gallimard, Tel, 1989, p. 22.

${ }^{27}$ Si le concept de champ a pu être importé avec succès en sociologie, c'est peut-être parce qu'il évoque l'idée d'opposition, qui correspond à la fois aux opérations usuelles de l'invention et de la rhétorique dissertatives (couples d'oppositions, thèse et antithèse, conciliation des contraires, renversement du pour au contre) et à un parti-pris idéologique (vision conflictuelle de la société). 
«Structure» veut dire «organisation », et ne dit rien de plus, si ce n'est, peut-être, organisation particulièrement cohérente, degré supérieur d'organisation; de même «champ» est mis pour «milieu» ou pour «groupe». Cette substitution donne l'illusion de passer à un niveau supérieur d'abstraction et de généralisation; mais «structure» désigne n'importe quelle organisation, de même que «champ » désigne n'importe quel groupe. Comme tout est organisation, que tout est à des degrés divers organisé, organisation étant plus ou moins synonyme d'ordre, on peut voir des structures partout, et donc prétendre expliquer tout par la structure. De même on peut tout expliquer par le «champ», qui peut désigner aussi bien la société dans son ensemble (le «champ social» chez Lewin), que des groupes plus ou moins restreints, des «milieux», des domaines d'activité, ou des domaines d'études (champ politique, champ intellectuel, champ du pouvoir, champ religieux, champ artistique, etc. $)^{28}$.

Plus ces formules sont vides, plus elles sont vagues, et plus nombreux sont les faits qu'elles évoquent et qui se transforment ainsi en autant de signes; c'est parce qu'on ne sait pas et qu'on ne peut savoir ce que l'on entend au juste par modernité que l'on peut voir partout des signes de modernité. C'est ce qui arrive lorsque le sociologue, au lieu de monter ses concepts à partir de l'observation et de théoriser progressivement, demande à un semblant d'ethnographie de confirmer et de soutenir une vision générale de la société, comme la «théorie de la domination»; dès lors qu'on essaie «de tout expliquer à partir de la base très étroite » de l'opposition entre dominants et dominés, on a toutes les chances de voir dans toute organisation, plus particulièrement dans toute organisation de l'espace, une expression de l'ordre social ${ }^{29}$. L'observation se trouve ainsi mise au service de l'interprétation, ou plutôt, plus insidieusement, l'interprétation contamine l'observation. L'effet de réel procuré par la mention évocatrice d'objets concrets, désignés par

28 «Structure» et «champ» sont spontanément associés; parler de «structure du champ» donne l'impression de s'élever à un niveau de pensée encore plus général et plus théorique. L'importation en sociologie du terme de champ s'inscrit dans le contexte du structuralisme; comme celui de la notion de structure, son emprunt aux sciences de la nature se rattache dès l'origine à une conception légaliste, logiciste et anti-historique de la causalité. Sur l'anti-historicisme du structuralisme anthropologique, voir infra, chap. III, 5. «S'appuyant sur le développement de la théorie des champs en physique, il [Lewin] a cherché à remplacer la causalité historique, qui explique un fait par des faits antérieurs, par une causalité dynamique, qui explique un fait par d'autres faits simultanés et organisés selon des rapports de force». D. Baillergeau, Blog ART / Champ social.

${ }^{29}$ C'est le cas, entre quantité d'exemples, quand on veut voir dans la disposition des instruments de l'orchestre sur le plateau l'expression d'une hiérarchie où les cordes, au premier plan, et plus particulièrement les violons, aristocratiques ou bourgeois, dominent les vents, populaires. Cette thèse s'auto-immunise d'emblée en négligeant les faits techniques et historiques qui la contrarient. Le préjugé interprétatif qui l'anime interdit de savoir si, jusqu'à quel point les places respectives des familles d'instruments sur le plateau de l'orchestre résultent de contraintes acoustiques, et par quels processus une hiérarchie sociale des instrumentistes a pu éventuellement venir se greffer sur ces contraintes; il oblige à oublier l'abondance de la littérature baroque et classique pour instruments à vents (les fanfares de Haendel et de Bach, les concertos pour trompette, pour cor, pour hautbois de Mozart et de Haydn, les sonates pour flûte de Bach, le concerto et le quintette pour clarinette de Mozart, etc.), à oublier le prestige de la flûte (jouée par Frédéric II), le statut de la clarinette (instrument emblématique de la musique maçonnique), à oublier aussi les violons, les violonistes et les musiques pour violon populaires, les violons juif, irlandais, tzigane, du Hardanger, les violoneux, les fiddles et les fiddlers. 
leurs noms d'usage, rangés dans des catégories indigènes pratiques, donne une impression de confrontation avec la réalité, de description objective, qui apporte de la consistance et de la vraisemblance à la thèse. Mais par ailleurs, l'idée dont on part sert constamment de grille de lecture, de déchiffrement de la réalité. Chacun des éléments qu'on fait entrer dans la description, chacun des faits, des traits et des relations qui la composent sont considérés comme des indicateurs, comme des signes qui «renvoient», par delà les apparences, à une structure cachée, à un système d'oppositions sous-jacent. Dans la mesure où les faits retenus et invoqués à l'appui de la théorie tirent leur signification de ce système, celle-ci ne peut qu'être confirmée par eux, et jamais démentie. Ce circulus herméneutique dispense et empêche de poser les questions capables de lever la suspicion qui tient lieu de démonstration et qu'il suffit de laisser planer pour emporter la conviction. Plus l'idée de départ est abstraite et générale, plus elle semble fondamentale, et plus ce cercle est difficile à rompre. C'est le cas, par exemple, quand le schème sociologique de la domination renvoie lui-même à un schème philosophique plus «profond», comme le dualisme de l'âme et du corps. Dès lors que les dominés sont aux dominants ce que le corps est à l'âme, ou ce que la matière est à l'esprit (sans doute aussi ce que la nature est à la culture), les pratiques populaires, ou réputées telles, se retrouvent automatiquement du côté du corps et de la matière; ces notions sont suffisamment vagues pour laisser le champ libre à la fantaisie interprétative de l'observateur, qui peut voir partout des indices d'un rapport au corps plus ou moins direct ou de goûts plus ou moins «matériels », et suffisamment riches en connotations pour permettre la réintroduction subreptice de jugements de goûts et de valeurs. Les pseudo-théories paraissent ainsi participer de l'infaillibilité idéale des théories universelles, alors qu'elles se réduisent à une herméneutique qui les rend irréfutables.

\section{LA RECHERCHE DES FAITS CONTRARIANTS}

Pour échapper à la dérive herméneutique, il faut donc définir les concepts qu'on met en œuvre par les éléments de réalité auxquels ils se rapportent et qu'ils désignent, et non par rapport à d'autres concepts comme y poussent l'héritage scolastique et la pratique de la dissertation (Mills 1967) ${ }^{30}$. C'est ainsi que Georges Dumézil protège la notion de triade contre la monomanie interprétative en précisant à la fois de quoi se composent les triades tri-fonctionnelles (qui sont des structures) et en quoi elles consistent:

30 «Quand nous envisageons ce que représente un mot, nous faisons de la sémantique; quand nous envisageons nos rapports avec d'autres mots, nous faisons de la syntaxe. Si j'introduis ces notions, c'est pour arriver à la formule lapidaire suivante: les suprêmes-théoriciens s'enivrent de syntaxe et n'entendent rien à la sémantique. Ils ne comprennent pas vraiment qu'en définissant un mot, nous encourageons simplement les autres à l'utiliser selon nos vœux; que le but d'une définition est de faire porter la discussion sur les faits, et que la définition a rempli son rôle si elle transforme un accord sur la forme en désaccord sur le fond, donnant ainsi le champ à de nouvelles recherches ». C. W. Mills, L'imagination sociologique, Paris, Maspéro, 1967, p. 38. L'opposition entre syntaxe et sémantique recouvre l'opposition entre théories différentielles et théories référentielles de la signification des mots. Cf. N. Wiley, «Inner Speech as a Language: A Saussurean Inquiry », Journal for the Theory of Social Behaviour, Vol. 36, n 3, Septembre 2006, pp. 319-341. 
Je voudrais sonner l'alarme. Depuis que mon travail a eu un peu d'audience, des personnes de bonne volonté, des jeunes, des moins jeunes, se sont mises à chasser les triades tri-fonctionnelles. Il ne se passe pas de mois sans que j'en reçoive de nouvelles, et il s'en publie, non seulement en France mais en Amérique. On m’a déjà proposé sept interprétations tri-fonctionnelles de l'intrigue, du personnel de l'Iliade. Or, parmi ces propositions, il y en a d'excellentes. De jeunes comparatistes d'avenir ont commencé à les publier; je les y encourage. Mais la grande majorité de ces propositions ne résiste pas à l'examen; ou bien les interprétations des termes sont forcées, ou bien il n'y a même pas de triade. Et cela m'amène à définir les conditions, disons les conditions minimales qui doivent être assurées, sauf justification explicite, pour que l'interprétation fonctionnelle d'une triade soit plausible, ou probable. D'abord, il faut qu'il y ait triade, c'est-à-dire qu'il y ait trois termes à la fois distincts, solidaires et exhaustifs, et qui soient rapprochés, présentés par les usagers eux-mêmes comme une triade. D'ailleurs il ne faut pas prendre un même terme pour représenter de façon confuse deux options. Il ne faut pas prendre trois termes arbitrairement dans une liste de sept ou huit. Et il faut qu'il y ait un élément d'unité, par exemple ce sera trois actions d'un même personnage, ou bien ce sera trois actions parallèles faites par trois personnages. Deuxième condition, il faut que l'interprétation fonctionnelle ne soit pas a priori invraisemblable, impossible; par exemple quelqu'un proposait de dire que dans la structure de l'indo-européen le fonctionnement du substantif était de la première fonction, le verbe, essentiellement le verbe d'action était de deuxième fonction et l'ensemble du vocabulaire de troisième. C'est absurde. En troisième lieu, il faut que les termes soient homogènes, c'est-à-dire qu'il s'agisse par exemple de trois dieux, et non de deux dieux et d'un animal, de trois scènes de même niveau littéraire, de trois objets ou de trois conduites, et non pas d'une conduite et de deux objets. Et, en dernier lieu, il faut que ces interprétations soient, le mot est ambitieux mais il faut le prononcer, il faut qu'elles soient évidentes, c'est-à-dire que au moins pour deux des termes l'interprétation fonctionnelle soit immédiatement claire et pour le troisième qu'elle soit rendue probable par une démonstration simple, courte et solide (Dumézil 2002) (1. $^{31}$

Il faut en conséquence bannir les concepts extra ou méta empiriques, impossibles à définir par rapport à des réalités constatables, les notions faussement générales, capables de s'appliquer indifféremment à des réalités différentes, les concepts métaphysiques, les concepts d'essence, bref tous les concepts qui, n'étant pas «de l'ordre du phénoménal»n'ont pas de place dans la science ${ }^{32}$. Mais, comme on l'a vu, il n'est pas facile de séparer les concepts indéfinissables des concepts peu ou mal définis, mais qui pourraient l'être. Pour distinguer entre les généralités sans contenu, qu'il faut rejeter d'emblée, et les formulations qui peuvent effectivement servir à classer et à comparer des faits, voire à définir des «espèces» de sociétés ou de cultures, il faut se demander si l'attribut qu'elles retiennent correspond à des traits spécifiques observables dans la réalité. On écartera les formules qui perdent leur intérêt et leur charme dès qu'on leur ajoute un déterminant (abondance de quoi? consommation de quoi?) On peut en revanche mettre à l'épreuve des formules mal définies, mais définissables; on se demandera par exemple en quoi les «sociétés rurales» se distinguent des «sociétés paysannes » ou des «sociétés urbaines», ce que l'on entend par «ville» et par prépondérance, ou domination de la ville, dans quelle mesure «société » désigne

${ }^{31}$ G. Dumézil, Entretiens avec Ollender, rediffusion par France-Culture, «les Chemins de la Connaissance», 30/01/02.

${ }^{32}$ Cf. R. Aron, Les grandes doctrines de sociologie historique, op. cit., t. II, p.107. 
alors la société dans son ensemble ou certains de ses éléments, comme les «sociétés villageoises ». De même on peut essayer de déterminer les caractéristiques qui distinguent les «sociétés industrielles » des sociétés pré-industrielles, agricoles, commerciales, manufacturières, artisanales, etc. ${ }^{33}$ On s'appuiera plus sûrement sur une notion telle que «société de castes », qui fait bien voir l'opposition entre types et pseudo-types ${ }^{34}$. En effet, la caste est à la fois une stylisation de la réalité, un cas limite, un type idéal à partir duquel on se propose de définir, par comparaison, d'autres formes élémentaires de la vie sociale et une réalité concrète, dont l'existence s'impose à l'observateur et à l'historien, qui se manifeste et se matérialise dans les interdits auxquels elle contraint les individus dans les domaines les plus divers de leur vie (Bouglé 1927, Dumont 1967) ${ }^{35}$.

Contre l'esprit de système, qui va jusqu'à inverser le sens des faits qui le contrarient (en voyant par exemple dans les manquements à une norme dominante ou légitime autant d'expressions indirectes, «déguisées », « déniées », c'est-à-dire autant de signes de la reconnaissance de cette norme, autant «d'hommages que le vice rend à la vertu»), il faut cultiver l'esprit de contradiction. A la convocation des exemples qui témoignent en faveur d'une thèse, on opposera la recherche méthodique des faits contrariants capables de la démentir. Il faut pour cela commencer par se débarrasser du discrédit qui s'attache aux notions de constat, de fait brut, et plus généralement, à la notion de fait elle-même. Sans doute, comme le remarquait déjà Simiand, "il n'y a pas de constatation qui ne soit déjà un choix» (Simiand 1903) ${ }^{36}$. La démarche scientifique repose en effet sur une première mise en forme, sur une simplification préliminaire qui ne retient de la réalité que les aspects auxquels l'état du savoir, c'est-à-dire de la question, conduit à s'intéresser (Poincaré 1927) ${ }^{37}$. Cette modélisation initiale est une opération déci-

${ }^{33}$ Voir, à propos de la «société industrielle» de Spencer, E. Durkheim, De la division du travail social, op. cit., p. 18.

${ }^{34}$ Expression d'une subjectivité interprétative plus ou moins travaillée (vision ou lubie, parti-pris, humeur ou caprice), les pseudo-types s'apparentent à des types construits dans la mesure où ils évoquent des contre-types, qui paraissent correspondre aux sociétés ou aux cultures d'où le trait prétendument typique est absent ; si l'on ne voit guère à quoi s'oppose la « société victimaire », on peut toujours opposer, du moins formellement, la société de consommation à la société de non-consommation, la société d'abondance à la société de pénurie, la société post-moderne aux sociétés modernes ou pré-modernes, la «culture de masse» à la «culture de l'élite», etc. La sociologie, l'anthropologie, l'histoire, où l'élaboration des types les plus généraux joue un rôle décisif sont particulièrement exposées à cette confusion (cf. infra, chap. III, 4).

${ }^{35}$ Cf. C. Bouglé, Essais sur le régime des castes, Paris, Alcan, 1927, notamment l'introduction, «Essence et réalité du régime des castes»; L. Dumont, Homo hierarchicus, essai sur le système des castes, Paris, Gallimard, 1967.

${ }^{36}$ «Contrairement à une apparence superficielle, en toute science, il n’y a pas de constatation qui ne soit déjà un choix, il n'y a pas d'observation qui ne présuppose quelque idée, quelque vue de l'esprit; il n'est pas de groupement de faits qui n'implique chez l'auteur du groupement (avec conscience nette ou conscience obscure) une certaine hypothèse constructive, une certaine préformation de science. Dans l'œuvre scientifique, la pensée qui conçoit et l'attention qui observe sont sans cesse en étroit commerce, travaillent ensemble, ne font qu'un». F. Simiand, «Méthode historique et sciences sociales », Revue de Synthèse Historique, 1903 (in F. Simiand, Méthode historique et sciences sociales, choix et présentation de M. Cedronio, Paris, Editions des archives contemporaines, 1987, pp. 159 sq.).

37 «Le savant intervient activement en choisissant les faits qui méritent d'être observés. Un fait isolé n'a par lui-même aucun intérêt; il en prend un si on a lieu de penser qu'il pourra aider à en prédire 
sive et risquée; alors qu'elle détermine toute la suite des observations et des raisonnements, elle peut résulter de partis-pris interprétatifs plus ou moins conscients qui ne retiennent d'emblée que les éléments de la réalité qui leur conviennent. Le sentiment de cet arbitraire, particulièrement fréquent dans les sciences de l'homme, a sans doute contribué à l'inversion relativiste du constat épistémologique de Simiand. De ce que les faits, pour être constatés, doivent faire l'objet d'une construction préalable, on passe à l'idée que les faits n'existent pas en eux-mêmes, en dehors et indépendamment de la relation que l'observateur entretient avec eux, et on revient pour finir à l'idée qu'ils n'existent que dans la conscience et par la volonté du sujet qui les observe et les interprète. A l'appui de cette thèse, on fait remarquer que cette construction préalable concerne aussi bien la représentation artistique que la représentation scientifique de la réalité; on en conclut que le point de vue du savant n'est pas moins subjectif, pas moins arbitraire, que celui du peintre ou du poète. Mais la conduite de la vie quotidienne repose elle aussi sur une modélisation initiale, sur la simplification du monde dans lequel nous vivons, sur une sélection des aspects sous lesquels nous l'envisageons, des objets dont il se compose et des événements qui s'y produisent; la prime éducation consiste essentiellement à apprendre à faire attention, c'est-à-dire à apprendre à quoi il faut faire attention. Cette modélisation pratique, dont dépendent l'adaptation et parfois la survie de l'individu, n'a rien d'arbitraire; elle rencontre constamment la résistance et les sanctions de la réalité.

Il faut donc se souvenir que si le point de vue fait l'objet, c'est aussi l'objet qui fait, ou du moins qui contraint le point de vue. Bien qu'il opte pour l'instrumentalisme et non pour le réalisme ${ }^{38}$, Poincaré distingue nettement entre la convention et le fait: "L'énoncé d'un fait est toujours vérifiable et pour la vérification nous avons recours soit au témoignage de nos sens, soit au souvenir de ce témoignage. C'est là proprement ce qui caractérise un fait. Si vous me posez la question: tel fait est-il vrai? je commencerai par vous demander, s'il y a lieu, de préciser les conventions, par vous demander, en d'autres termes, quelle langue vous avez parlé; puis, une fois fixé sur ce point, j'interrogerai mes sens et je répondrai, oui ou non (...) si nous pouvons dire que le fait en question est faux, n'est-ce pas justement parce qu'il n'est pas une création libre et arbitraire de notre esprit, une convention déguisée, auquel cas il ne serait ni vrai ni faux?» (Poincaré 1927: $233)^{39}$. A l'arbitraire des choix subjectifs, à la présomption interprétative des

d'autres; ou bien encore si, ayant été prédit, sa vérification est la confirmation d'une loi. Qui choisira les faits qui, répondant à ces conditions, méritent le droit de cité dans la science ? C'est la libre activité du savant». H. Poincaré, La valeur de la science, Paris, Flammarion, 1927, p. 233.

38 Voir infra, chap. III, 1.

39 «Quand je dis, par exemple, l'unité de longueur est le mètre, c'est un décret que je porte, ce n'est pas une constatation qui s'impose à moi. Il en est de même (...) quand il s'agit par exemple du postulatum d'Euclide (...) Les faits sont classés en catégories, et si l'on me demande si le fait que je constate rentre ou ne rentre pas dans telle catégorie, je n'hésiterai pas.

Sans doute cette classification comporte assez d'arbitraire pour laisser à la liberté ou au caprice de l'homme une large part. En un mot, cette classification est une convention. Cette convention étant donnée, si l'on me demande: tel fait est-il vrai? je saurai toujours que répondre, et ma réponse me sera imposée par le témoignage de mes sens. Si donc pendant une éclipse, on demande: fait-il noir? tout le monde répondra oui. (...) De même, en mathématiques, quand j'ai posé les définitions et les postulats 
systèmes de pensée, on peut et on doit donc opposer des evidences, c'est-à-dire des faits dûment constatés, bien établis, clairement et précisément caractérisés (notamment par des propriétés mesurables, définies au moyen de catégories et de critères universels, comme les caractéristiques techniques).

Le fait contrariant par excellence est le fait décisif qui suffit à lui seul, par sa présence ou par son absence, à infirmer une hypothèse. Les propositions théoriques, dans les sciences de l'homme, s'exposent plus rarement que dans les autres sciences de l'observation à cette réfutation sans appel. En voulant à tout prix leur opposer un fait crucial, on risque même de présenter une objection naïve, préscientifique. C'est ce qui arrive quand on oppose des cas individuels à un raisonnement statistique, par exemple lorsqu'on invoque des réussites universitaires improbables (filles d'ouvriers admises à l'Ecole polytechnique) pour contrer la thèse de l'inégalité sociale des chances d'accès au niveau supérieur des études supérieures. La présentation de ces exceptions, incapables d'infirmer une régularité statistique par définition probabiliste, se réfère elle aussi à l'épistémologie sous-jacente du déterminisme strict. Pour éprouver une thèse, on est donc conduit le plus souvent à rassembler une série de faits qui la contredisent; non seulement les faits qui lui échappent, qu'elle ne parvient pas à expliquer et à inclure, et qui marquent ses limites, restreignent sa portée, mais aussi et surtout les faits qu'elle ne peut admettre, dont elle doit exclure l'existence, et dont l'accumulation, et plus encore la convergence, obligent à la rejeter. Il arrive pourtant qu'un seul fait contrariant suffise à ruiner une conjecture. Il faut pour cela que celle-ci, conformément à la définition de la science logico-expérimentale de Pareto, soit un raisonnement élaboré à partir de l'observation de la réalité, comme c'est le cas en matière de classement, quand l'attribution d'un spécimen à une catégorie met à l'épreuve la pertinence de celle-ci et la manière dont elle a été constituée. C'est ainsi, par exemple, qu'E. Benveniste teste la définition «structurelle» ou «typologique» des langues indo-européennes proposée par N. Troubetzkoy, par opposition à la définition "génétique» ou «historique», qui «distribue les langues en familles supposées issues d'un prototype commun» (Benveniste 1993 [1966]: $99)^{40}$. D'après Troubetzkoy, on reconnaît qu'une langue est indo-européenne à ce qu'elle présente la réunion de six traits pertinents, eux-mêmes définis au moyen des catégories et des concepts propres à la linguistique et formulés dans son vocabulaire technique spécialisé ${ }^{41}$. Benveniste pose alors «une question de fait», à

qui sont des conventions, un théorème ne peut plus être que vrai ou faux. Mais, pour répondre à cette question: ce théorème est-il vrai? ce n'est plus au témoignage de mes sens que j'aurai recours, mais bien au raisonnement». H. Poincaré, «Le fait brut et le fait scientifique», in La valeur de la science, op. cit., pp. 220 sq. La notion de «convention», actuellement très en vogue dans les sciences de l'homme, est devenue la notion de base du relativisme. C'était déjà le cas chez Edouard Le Roy, catholique militant, successeur de Bergson au Collège de France et à l'Académie Française, dont Poincaré rejette l'anti-intellectualisme: (pour Le Roy) «la Science n'est faite que de conventions, et c'est uniquement à cette circonstance qu'elle doit son apparente certitude; les faits scientifiques, et à fortiori, les lois sont l'œuvre artificielle du savant; la science ne peut donc rien nous apprendre de la vérité, elle ne peut nous servir que de règle d'action.»Ibid, p. 214.

${ }^{40}$ E. Benveniste, Problèmes de linguistique générale, 1, Paris, Gallimard, 1993 [1966], p. 99.

41 1. Il n'y a pas d'harmonie vocalique; 2 . Le consonantisme de l'initiale n'est pas plus pauvre que celui de l'intérieur ou de la finale; 3 . Le mot ne doit pas nécessairement commencer par la racine; 
laquelle «il sera répondu affirmativement si et seulement si aucune autre famille linguistique ne possède les six caractères énoncés par Troubetzkoy comme propres aux langues indo-européennes ». Pour répondre à cette question, Benveniste prend «au hasard un spécimen d'une langue sûrement non indo-européenne» (en fait une langue dont on a tout lieu de penser, étant donné sa localisation, qu'elle n'est pas indo-européenne), et pour laquelle on dispose «d'une description excellente et aisément accessible» (le takelma, langue indienne de l'Oregon, étudiée par E. Sapir). Benveniste constate que «le takelma possède ensemble les six traits dont la réunion constituait, aux yeux de Troubetzkoy, la marque distinctive du type indo-européen», suppose «qu'une enquête identique ferait rencontrer des cas analogues dans d'autres familles», et conclut «que la définition proposée par Troubetzkoy est en tout cas réfutée par les faits ${ }^{42}$.

4. Les formes ne sont pas constituées seulement par des affixes, mais aussi par des alternances vocaliques à l'intérieur des morphèmes radicaux; 5. Outre les alternances vocaliques, les alternances consonantiques libres jouent aussi un rôle morphologique; 6. Le sujet d'un verbe transitif est traité comme le sujet d'un verbe intransitif. E. Benveniste, op. cit., p. 108.

${ }^{42}$ E. Benveniste, op. cit., p. 109. 